\title{
Effects of Cangfudaotan Tang on Expression of Organic Anion Transporting Polypeptide (oatp2b1) in Liver and Kidney Tissues of Rats with Phlegm Dampness Type Polycystic Ovary Syndrome (PCOS)
}

\author{
Ke Chen, Aizhen Pan*, Jianjun Li, Kefang Chen, Xiangping Hou \\ Department of Traditional Chinese Medicine, Sun Yat-Sen Memory Hospital of Sun Yat-sen University, Guangzhou, China \\ Email: *China.keshan333@163.com
}

How to cite this paper: Chen, K., Pan, A.Z., Li, J.J., Chen, K.F. and Hou, X.P. (2016) Effects of Cangfudaotan Tang on Expression of Organic Anion Transporting Polypeptide (oatp2b1) in Liver and Kidney Tissues of Rats with Phlegm Dampness Type Polycystic Ovary Syndrome (PCOS). Chinese Medicine, 7, 166-174. http://dx.doi.org/10.4236/cm.2016.74016

Received: November 16, 2016

Accepted: December 16, 2016

Published: December 20, 2016

Copyright $\odot 2016$ by authors and Scientific Research Publishing Inc. This work is licensed under the Creative Commons Attribution International License (CC BY 4.0).

http://creativecommons.org/licenses/by/4.0/

\section{(c) (i) Open Access}

\section{Abstract}

Objective: To explore the effect of Cangfudaotan Tang on phlegm dampness type of PCOS and the role of oatp2b1 in transportation and transformation of phlegm dampness. Methods: 36 SD female rats were randomly divided into three groups: blank control group, model group and Cangfudaotan Tang group, 12 cases in each one. After PCOS rat models were made, rats of Cangfudaotan Tang group were treated with Cangfudaotan Tang $(1.42 \mathrm{~g} / \mathrm{kg} / \mathrm{d})$ by intragastric administration for 14 days; blank control and model group were given with isodose saline. The expression of oatp2b1 mRNA/Protein in liver and kidney tissues was measured and the level of testosterone $(\mathrm{T})$, follicle stimulating hormone $(\mathrm{FSH})$, estradiol $\left(\mathrm{E}_{2}\right)$, luteinizing hormone(LH), Serum total cholesterol (TG), Triacylglycerols (TC), high density lipoprotein cholesterol (HDL-C) and low density lipoprotein cholesterol (LDL-C) were detected at the same time. Results: Compared with blank control group, the expression of oatp2b1 mRNA and the level of TC, TG, LDL, LH, FSH, T in model group were significantly increased $(\mathrm{P}<0.05)$, while the level of HDL was significantly decreased $(\mathrm{P}<0.05)$; compared with model group, the expression of oatp2b1 mRNA and the level of TC, TG, LDL in Cangfudaotan Tang group were significantly lowered $(\mathrm{P}<0.05)$; the level of HDL was significantly higher; the oatp2b1 protein in kidney and liver tissues had different degrees of expression, while there was no statistical significance among the three groups. Conclusions: Oatp2b1 might be one of the material bases participating in transportation and transformation of phlegmy dampness. The mechanism of Cangfudaotan Tang treating phlegm dampness type of PCOS may be achieved by regulating the expres- 
sion of oatp2b1.

\section{Keywords}

Cangfudaotan Tang, Polycystic Ovary Syndrome, Phlegm Dampness, Organic Anion Transporting Polypeptide, Oatp2b1

\section{Introduction}

With the human living standards rising, hyperlipidemia and other related diseases caused by high fat food intake have become a major disease. Chinese medicine believes that high fat diet is the main cause of phlegm dampness syndrome and hyperlipidemia belongs to phlegm turbidity of the traditional Chinese medicine, which provides a theoretical basis for rat model of phlegm dampness syndrome established by the high fat diet. Research indicates, organic anion transporter polypeptide (oatp) can efficiently transport a variety of foreign organisms, which plays an important role in transportation and transformation of phlegm dampness [1]. Phlegm dampness type is one of the most common syndrome types of PCOS. Cangfudaotan Tang has the effect of eliminating dampness and resolving phlegm, which is one of effective prescriptions in treating phlegm dampness type of PCOS. This study selected oatp2b1, which is widely distributed in various tissues of animal, as the research object. The aim of the study was to explore the effect of Cangfudaotan Tang on phlegm dampness type of PCOS and the role of oatp2b1 in transportation and transformation of phlegm dampness, by observing the expression of oatp2b1 in rat liver and kidney tissues with phlegm dampness type of PCOS. The findings of the report are as follows.

\section{Material and Instruments}

\subsection{Animals}

36 six-week-old Female SD rats of SPF level, weighing 35 - $45 \mathrm{~g}$, were provided by the animals central of Guangdong province, License number is 0003459, Guangdong License number is 20030001 .

\subsection{Drugs and Reagents}

Herbal medicine constituting Cangfudaotan Tang were provided by dispensary of traditional Chinese medicine in Sun Yat-Sen memory hospital of Sun Yat-sen University; Human Chorionic Gonadotropin (Shanghai Yili Biological Technology Co. Ltd.); Sodium Prasterone Sulfate (Jiangsu Huanlian Pharmaceutical Co., Ltd.); RT-PCR kit: $\beta$-actin (mouse) monoclonal antibody and OATP-B (SANTA CRUZ); Anti-goat IgG-HRP (ZSGB-BIO); RNAiso ${ }^{\text {ra }}$ Plus extraction kit (TaKaRa, No.D9108A); $5 \times$ quantitative PCR buffer, $5 \times$ RT buffer, MMLV, dNTPs, Taq enzyme (Guangzhou Huayin Medical Technology Co, Ltd. No. 871340); OligoT (Promega); ELISA kit (Guangzhou yale pharmaceutical company). 


\subsection{Instruments}

3K15 desktop high-speed refrigerated centrifuge machines (Sigma); PTC-150 automatic fluorescence quantitative PCR instrument (MJ Opticon2); MDF-382EN ultra low temperature refrigerator (Sanyo); BS224S electronic balance (Sartorius), 164-5056 high voltage electrophoresis apparatus (BoLe).

\section{Methods}

\subsection{Animal Group and Intervention Methods}

36 rats were randomly divided into three groups: blank control group, model group and Cangfudaotan Tang group, 12 cases in each one. Blank control group: Conventional diet and natural environment, free eating and drinking water. Rats of model and Cangfudaotan Tang group were fed with high fat diet in the natural environment. (The high fat feed was purchased from the animals central of Guangdong province, and its components were as follows: basic feed $55 \%$, lard $15 \%$, sucrose $21 \%$, egg yolk powder $9 \%$, cholate $0.5 \%$.) Meanwhile, reference to the Luoshi method to establish PCOS rat models [2]. Rats of above two groups were injected with Sodium Prasterone Sulfate (9 $\mathrm{mg} / 100 \mathrm{~g} / \mathrm{d}$ ) for 40 days. On sixth days, rats were simultaneously given human chorionic gonadotropin (HCG) at dose of 1.5 units by subcutaneous injection for 35 days. After phlegm dampness of PCOS rat models were successfully made, rats of Cangfudaotan Tang group were treated with Cangfudaotan Tang $(1.42 \mathrm{~g} / \mathrm{kg} / \mathrm{d})$ by intragastric administration, and blank control and model group were given with isodose saline. Cangfudaotan comes from "Tianshi Ye for diagnosis and treatment of Gynecological diseases", and it consists of Poria $15 \mathrm{~g}$, Rhizoma atractylodis $10 \mathrm{~g}$, Fabanxia $10 \mathrm{~g}$, Fructus Ponciri 10 g, Rhizoma cyperi 10 g, Pericarpium citri nobilis 6 g, Arisaematis Rhizoma 6 g, Ginger 6 g, Radix glycyrrhizae 6 g. Rats of model group and blank control group continue to be fed with high fat diet and conventional diet respectively.

\subsection{Diagnostic Criteria of Phlegm Dampness Constitution in Rats [3] [4]}

Obesity, decreased activity, decreased appetite, dull and fluffy coat, loose stools, decreased reaction ability, decreased water intake.

\subsection{Index Detection and Methods}

\subsubsection{Specimen Collection}

Anesthesia was performed with $3 \%$ pentobarbital sodium $(2 \mathrm{ml} / \mathrm{kg}$. b.w $)$ by the way of intraperitoneal injection in rats, each piece of tissue was drawn from liver and kidney, each size of sample was about $5 \mathrm{~mm} \times 5 \mathrm{~mm}$, putted samples into the freezing tube. Within 15 minutes, all samples were preserved into the $-80^{\circ} \mathrm{C}$ refrigerator.

\subsubsection{The Expression of oatp2b1 mRNA Detection}

Real-time fluorescent quantitative PCR method. Design and synthesis of primers: to find the mRNA of rats OATP2b1 and GAPDH on the GenBank; the specific primer probes were designed by Primer express 2.0 software; instruments for the ABI 3900 
desktop high throughput DNA synthesizer. The primer sequences are shown in Table 1. The RT-PCR cycling conditions: $94^{\circ} \mathrm{C}, 20 \mathrm{~s} ; 94^{\circ} \mathrm{C}, 5 \mathrm{~s}, 60^{\circ} \mathrm{C}, 30 \mathrm{~s}$, read FAM fluorescent value, 40 cycle.

\subsubsection{The Protein Expression Level of oatp2b1 Determination}

Using Western Blot method. Protein were extracted from liver and kidney (keygen protein extraction kit), and then tissues homogenate, ultracentrifugation $\left(4^{\circ} \mathrm{C}, 10000\right.$ $\mathrm{r} / \mathrm{min}, 5 \mathrm{~min}$ ), 60 minutes membrane proteins, SDS electrophoresis, transfer membrane, blocking, primary antibodies, secondary antibodies, ECL assay, the level of oatp2b1 protein expression was calculated according to $\mathrm{p}$-tublin 55ku reference value.

\subsubsection{Serum Lipids (TC, TG, LDL-C, HDL-C) Examination}

Serum lipid kit was purchased from Shanghai chemical reagent company of China Pharmaceutical Group (Batch number: 20110712). After the last treatment and then rats in each group were fasting for 16 hours, blood samples were obtained from abdominal aorta, ultracentrifugation ( $3000 \mathrm{r} / \mathrm{min}, 10 \mathrm{~min}$ ), serum collection, used to detect the level of TC, TG, LDL-C and HDL-C.

\subsubsection{Hormone Measurement (LH, FSH, T, $\mathrm{E}_{2}$ )}

The method of serum collection is the same as above and the level of $\mathrm{LH}, \mathrm{FSH}, \mathrm{T}$ and $\mathrm{E}_{2}$ were measured by ELISA.

\subsubsection{Statistical Analysis}

Application of statistical software SPSS 13.0 for analyzing the data; all data are expressed as means \pm standard deviation; comparisons among multiple groups means were analyzed by variance analysis $(\alpha=0.05)$; in the condition of refusing to obey normal distribution, comparisons among multiple groups were performed with KruskalWallis test $(\alpha=0.10)$; comparisons between any two groups were achieved by MannWhitney $\mathrm{U}$ test.

\section{Results}

\subsection{General Symptoms of Rats}

In the course of feeding, compared with blank control group, rats of high fat diet were

Table 1. The primer sequences of target gene.

\begin{tabular}{ll}
\hline Gene & Primer sequences \\
\hline OATP2b1 & forward: 5‘GAT AAG ACC CTG GCA GTG GGA3’ \\
& PROBE: FAM-CAA TTC ATG CTC CTG CGA GTG TTG GCC-BHQ1 \\
& reverse: 5‘GAT GGC ACT GCC ATG GAT CAC3’ \\
& forward: 5‘GAA GCT CAT TTC CTG GTA TGA CAA TG3’ \\
GAPDH & PROBE: FAM-TAT GGC TAC AGC AAC AGG GTG GTG GAC-BHQ1 \\
& reverse: 5‘CTT GGA GGC CAT GTA GGC CAT G3’
\end{tabular}


more docile, decreased activity, decreased reaction ability, dull and fluffy coat. After eight weeks, rats of model group and Cangfudaotan Tang group had these symptoms such as increased weight, decreased appetite, loose stools, decreased water intake, and so on.

\subsection{Effects of Cangfudaotan Tang on Expression of oatp2b1 mRNA in Rat Liver and Kidney Tissues (Table 2)}

Compared with blank control group, the expression of oatp2b1 mRNA in rat liver and kidney tissue of model group were significantly decreased $(\mathrm{P}<0.01, \mathrm{P}<0.05)$; Compared with model group, the expression of oatp2b1 mRNA in rat liver and kidney tissue of Cangfudaotan Tang group were significantly increased $(\mathrm{P}<0.01, \mathrm{P}<0.05)$.

\subsection{Effects of Cangfudaotan Tang on Protein Expression of oatp2b1 in Ratliver and Kidney Tissues (Figure 1, Table 3)}

The oatp2b1 protein in kidney and liver tissues had different degrees of expression, protein expression intensity: Cangfudaotan Tang group $>$ blank control group $>$ model group, however, there was no statistical significance among the three groups $(P>0.05)$, the reason may be associated with that the time of establishing the phlegm dampness type PCOS model is too short. In clinical, phlegm dampness type polycystic ovary syndrome is a chronic course of disease, which generally lasts for several months, years or even decades. However, the experimental model is an acute process. Although rats have clinical manifestations of PCOS, the changes in the microcirculation are not necessarily synchronized.

\subsection{Effects of Cangfudaotan Tang on Serum Lipid Levelin Rats (Table 4)}

After the final administration, serum levels of TC, TG, LDL, and HDL were measured for all rats and finally the changes were compared between the groups. Compared with blank control group, the level of TC, TG and LDL in model group were significantly increased $(\mathrm{P}<0.05)$ while the level of HDL was significantly decreased $(\mathrm{P}<0.05)$, suggesting that the high fat diet could lead to metabolic disorder of blood lipids and the phlegm-dampness rat model was successfully established. Cangfudaotan Tang resulted in significant reduction of TC, TG and LDL $(\mathrm{P}<0.05)$ as well as significant increase of HDL $(\mathrm{P}<0.05)$ compared to model group, which indicate that Cangfudaotan Tang has the function of regulating lipids.

Table 2. Effects of Cangfudaotan Tang on expression of oatp2b1 mRNA ( $n=12)$.

\begin{tabular}{ccc}
\hline Groups & Liver tissues & Kidney tissues \\
\hline Blank control group & $2.23 \pm 1.59$ & $2.52 \pm 1.84$ \\
Model group & $1.04 \pm 3.02^{* *}$ & $1.88 \pm 1.34^{*}$ \\
Cangfudaotan Tang group & $2.66 \pm 1.86^{*}$ & $5.27 \pm 4.79^{\# *}$ \\
\hline${ }^{*} \mathrm{P}<0.05,{ }^{* *} \mathrm{P}<0.01$ vs. blank control group ${ }^{*} \mathrm{P}<0.05,{ }^{* *} \mathrm{P}<0.01$ vs. model group. &
\end{tabular}



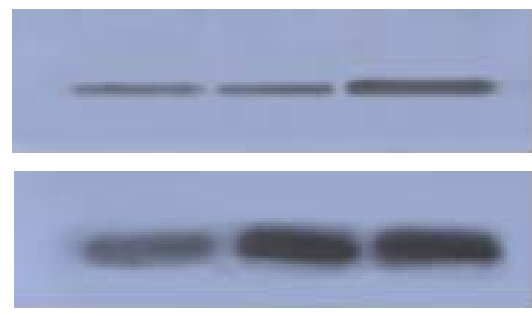

liver
A

C

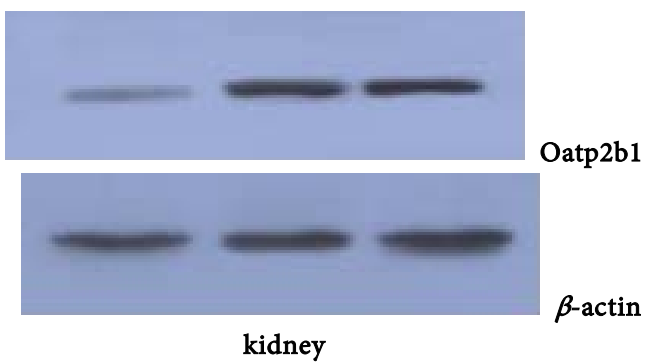

Figure 1. Effects of Cangfudaotan Tangon protein expression of oatp2b1. A. Blank control group. B. Model group. C. Cangfudaotan Tang group.

Table 3. Effects of Cangfudaotan Tang on protein expression of oatp2b1 $(n=12)$.

\begin{tabular}{ccc}
\hline Groups & Liver tissues & Kidney tissues \\
\hline Blank control group & $0.33 \pm 0.11$ & $0.25 \pm 0.08$ \\
Model group & $0.32 \pm 0.09$ & $0.24 \pm 0.06$ \\
Cangfudaotan Tang group & $0.33 \pm 0.12$ & $0.26 \pm 0.07$ \\
\hline
\end{tabular}

Table 4. Effects of Cangfudaotan Tang on serum lipid level $(\mathrm{mmol} / \mathrm{L}, \mathrm{n}=12)$.

\begin{tabular}{ccccc}
\hline Groups & TC & TG & LDL & HDL \\
\hline Blank control group & $2.05 \pm 0.43$ & $0.83 \pm 0.37$ & $1.22 \pm 0.34$ & $0.88 \pm 0.71$ \\
Model group & $4.85 \pm 0.94^{* *}$ & $1.51 \pm 0.46^{*}$ & $3.53 \pm 0.72^{* *}$ & $0.31 \pm 0.06^{*}$ \\
Cangfudaotan Tang group & $2.43 \pm 0.56^{\# \#}$ & $1.14 \pm 0.25^{\#}$ & $1.25 \pm 0.40^{\# \#}$ & $0.76 \pm 0.64^{\#}$ \\
\hline
\end{tabular}

${ }^{\star} \mathrm{P}<0.05,{ }^{\star} \mathrm{P}<0.01$ vs. blank control group; ${ }^{*} \mathrm{P}<0.05,{ }^{* *} \mathrm{P}<0.01$ vs. model group.

\subsection{Effects of Cangfudaotan Tang on Hormones Level in Rats (Table 5)}

Comparison of hormones level in three groups, the serum $\mathrm{T}$ and LH/FSH of model group were higher $(\mathrm{P}<0.01)$ and $\mathrm{E} 2$ was lower $(\mathrm{P}<0.01)$ than that of blank control group. After treatment, the level of serum T, LH and LH/FSH were decreased $(\mathrm{P}<$ $0.01)$.

\section{Discussion}

The emergence of phlegm dampness is related to improper diet, especially high-fat diet, viscera dysfunction. Above factors result in transport disorders of water and fluid, and then retention in vivo. In this study, the model of rat was induced by "high fat diet", which is closely related to the etiology of phlegm dampness syndrome. Liu Tao [4] research suggests that this model not only accord with the characteristic of phlegm dampness syndrome, but also has other typical features, such as obesity, hyperlipidemia, fatty liver, glucose tolerance and insulin resistance syndrome. Besides, high fat diet model has the pathological mechanism of hyperlipidemia, hemorheology and endothelia injury. Thus, recently, rat model induced by high fat diet is also used as the model for syndrome of blood stasis and phlegm dampness in Chinese medicine [5]. There are 
Table 5. Effects of Cangfudaotan Tang on hormones level.

\begin{tabular}{cccccc}
\hline Groups & LH $(\mathrm{mIU} / \mathrm{ml})$ & FSH $(\mathrm{mIU} / \mathrm{ml})$ & LH/FSH & T $(\mathrm{ng} / \mathrm{ml})$ & E2 $(\mathrm{pg} / \mathrm{ml})$ \\
\hline Blank control group & $23.67 \pm 4.62$ & $8.69 \pm 3.43$ & $1.94 \pm 2.42$ & $0.79 \pm 0.23$ & $23.36 \pm 3.51$ \\
Model group & $48.71 \pm 5.54^{\# \#}$ & $7.94 \pm 2.53^{\#}$ & $5.09 \pm 2.17^{\# \# *}$ & $1.72 \pm 0.69^{\# \# *}$ & $11.24 \pm 3.94^{\# \#}$ \\
Cangfudaotan Tang & $26.32 \pm 6.02^{*}$ & $8.03 \pm 3.71$ & $3.12 \pm 1.66^{* \#}$ & $0.47 \pm 0.26^{* \#}$ & $23.81 \pm 4.19^{*}$ \\
\hline
\end{tabular}

${ }^{*} \mathrm{P}<0.05,{ }^{* *} \mathrm{P}<0.01$ vs. blank control group; ${ }^{\#} \mathrm{P}<0.05,{ }^{* *} \mathrm{P}<0.01$ vs. model group.

a large number of "phlegm turbidity", "pathogenic damp" (food, metabolites, endogenous and exogenous toxins, drug metabolites, etc.) and other foreign organisms during the course of organism supersession [6]. Modern studies have indicated that oatp can efficiently transfer many foreign organisms and play a key role in the transport of phlegm dampness [7] [8]. Oatp2b1 is widely distributed in the intestine, kidney, lung, liver and other tissues and organs, as an important transporter of xenobiotics and endogenous substances, and the normal function of oapt plays an important part in maintaining balance between internal and external environment of body and cell [9] [10]. Oatp's dysfunction can cause dampness of the absorption, distribution and excretion of obstacles, a partial or whole body fluid balance disorders, leading to diseases [11] [12] [13]. Besides oatp2b1 also participates in the absorption and metabolism of estrogen in the intestinal tract. Sai Y. found that oatp2b1 was expressed in intestinal caco-2 cellsas, as a transporter of estrone metabolism, which can mediate estrone into cells [14]. Therefore, oatp can mediate the metabolism and absorption of reproductive endocrine hormone in the body, which influences the occurrence and development of PCOS in a certain extent.

The results of this study suggested that oatp2b1 mRNA in kidney and liver tissues of normal rat had different degrees of expression. After high fat diet for 3 months, the expression of oatp2b1 mRNA in rat liver and kidney tissue of model group was significantly decreased. Compared to blank control group, the level of TC, TG and LDL in model group were significantly increased $(P<0.05)$ while the level of HDL was significantly decreased $(\mathrm{P}<0.05)$, suggesting that the high fat diet could lead to metabolic disorder of blood lipids and the phlegm-dampness rat model was successfully established. According to the theory of traditional Chinese medicine, improper diet injury spleen, spleen deficiency leads to transportation and transformation disorder, retention of dampness, dampness condenses into phlegm, phlegm stagnates activities of qi, qi-stagnancy and blood stasis, blood running smooth, context blocked, finally, liver is damaged, prolonged diseases involve kidney. Because of liver dysfunction, the liver failing to dredge the spleen, dys-splenism result in retention of dampness, to form a pathological product of phlegm dampness. Zhang Jingyue said "phlegm dampness depends on transportation and transformation by spleen, the root of phlegm dampness lies in the kidney". The pathological products in turn can aggravate the liver and kidney dysfunction, reciprocal causation, forming a vicious spiral. Oatp2b1 as a membrane transport protein, which is advantageous to the absorption, distribution, utilization and 
elimination of xenobiotics and endogenous substances, especially drugs in the body [15]. The results of the study indicate that oatp2b1 plays an important role in the transport of phlegm dampness, and oatp $2 \mathrm{~b} 1$ might be one of the important material bases in water metabolism.

Spleen controlling digestion, one of functions is transmitting and distributing the fluids, which can regulate the balance of body fluid metabolism. Therefore, the spleen has a special sensitivity to dampness, traditional Chinese medicine called "the spleen liking dryness and disliking dampness". Spleen as the hub of water and fluid metabolism, which play an internuncial role. So tonifying spleen is key to transport of dampness. In the principle of treating disease from the root, the treatment group was treated with Cangfudaotan Tang, which has the function of fortifying the spleen and drying dampness. After 4 weeks of Cangfudaotan Tang treatment in Cangfudaotan Tang group, the results showed that the expression of oatp $2 \mathrm{~b} 1 \mathrm{mRNA}$ was significantly increased in liver and kidney tissue. It is demonstrated that Cangfudaotan Tang can improve the state of phlegm dampness by enhancing the excretion function of liver and kidney, so as to achieve the effect of treating phlegm dampness type of PCOS.

The oatp2b1 protein in kidney and liver tissues had different degrees of expression, and the intensity of protein expression was: Cangfudaotan Tang group > blank control group $>$ model group, however, there was no statistical significance among the three groups with statistical methods of Mann-Whitney $U$ ( $P$ > 0.05). This result might be related to the modeling method, protein sampling and specimen preparation and other factors, which is worth to be further verified.

\section{Source of Support}

This work was supported by the National Science Foundation for Young Scientists of China (Grant No. 81503606) \& Guangdong province administration of traditional Chinese medicine (NO: 201410480) and Guangdong province science and technology plan projects (NO: 2013B21800235).

\section{References}

[1] Zair, Z.M., Eloranta, J.J., Stieger, B., et al. (2008) Pharmacogenetics of OATP (SLC21/ SLCO) OAT and OC (SLC22) and PEPY (SLC15) Transporters in the Intestine, Liver and Kidney. Pharmacogenomics, 9, 597-624. https://doi.org/10.2217/14622416.9.5.597

[2] Zeng, L., Zhao, H.Y. and Luo, S.P. (2010) The Study of Improving rat Polycystic Ovarian Syndrome-Insulin Resistance (PCOS-IR) Model. CJTCMP, 5, 2341-2345.

[3] Tan, Z.H., Li, X.J., Wu, J., et al. (2008) Study on Phlegm Dampness Syndrome in TCM with Impaired Glucose Regulation. Journal of Bright Chinese Medicine, 23, 893-895.

[4] Liu, T., Xu, Q.L., Yang, S.Y., et al. (2009) Establishment and Evaluation of Animal Model of Phlegm Dampness Syndrome Induced by High Fat Diet. Journal of Changchun University of Traditional Chinese Medicine, 25, 333-334.

[5] Tian, J.Z., Wang, Y.Y., Xu, Y., et al. (2006) The Types, Evaluation and Research of Animal Model of Blood Stasis Syndrome. Journal of Beijing University of Traditional Chinese 
Medicine, 29, 396-400.

[6] Sarr, F.S., André, C. and Guillaume, Y.C. (2010) A Novel Biochromatographic Oatp2 Column to Study the Transmembrane Transport of Statins. Journal of Pharmaceutical and Biomedical Analysis, 52, 93-98. https://doi.org/10.1016/j.jpba.2009.11.029

[7] Hagenbuch, B. and Gui, C. (2008) Xenobiotic Transporters of the Human Organic Anion Transporting Polypeptides (OATP) Family. Xenobiotica, 38, 778-801. https://doi.org/10.1080/00498250801986951

[8] Wojtal, K.A., Eloranta, J.J., Hruz, P., Gutmann, H., Drewe, J., Staumann, A., Beglinger, C., Fried, M., Kullak-Ublic, G.A. and Vavricka, S.R. (2009) Changes in mRNA Expression Levels of Solute Carrier Transporters in Inflammatory Bowel Disease Patients. Drug Metabolism and Disposition, 37, 1871-1877. https://doi.org/10.1124/dmd.109.027367

[9] Noé, J., Portmann, R., Brun, M.E., et al. (2007) Substrate-Dependent Drug-Drug Interactions between Gemfibrozilfluvastatin and Other Organic Anion-Transporting Peptide (OATP) Substrates on OATP1B1, OATP2B1 and OATP1B3. Drug Metabolism and Disposition, 35, 1308-1314. https://doi.org/10.1124/dmd.106.012930

[10] Kalliokoski, A. and Niemi, M. (2009) Impact of OATP Transporters on Pharmacokinetics. British Journal of Pharmacology, 15, 693-705. https://doi.org/10.1111/j.1476-5381.2009.00430.x

[11] Hagenbuch, B. and Gui, C. (2008) Xenobiotic Transporters of the Human Organic Anion Transporting Polypeptides (OATP) Family. Xenobiotica, 38, 778-801.

[12] König, J., Seithel, A., Gradhand, U. and Fromm, M.F. (2006) Pharmacogenomics of Human OATP Transporters. Naunyn-Schmiedeberg's Archives of Pharmacology, 372, 432-443. https://doi.org/10.1007/s00210-006-0040-y

[13] Wojtal, K.A., Eloranta, J.J., Hruz, P., Gutmann, H., Drewe, J., Staumann, A., Beglinger, C., Fried, M., Kullak-Ublick, G.A. and Vavricka, S.R. (2009) Changes in mRNA Expression Levels of Solute Carrier Transporters in Inflammatory Bowel Disease Patients. Drug Metabolism and Disposition, 37, 1871-1877. https://doi.org/10.1124/dmd.109.027367

[14] Sai, Y., Kaneko, Y., Ito, S., et al. (2006) Predominant Contribution of Organic Anion Transport in Polypeptide oatp2b1 to Apical Uptake of Estrone-3-Sulfate by Human Intestinal Caco-2 Cells. Drug Metabolism and Disposition, 34, 1423-1431. https://doi.org/10.1124/dmd.106.009530

[15] Fuchikami, H., Satoh, H., Tsujimoto, M., et al. (2006) Effects of Herbal Extracts on the Function of Human Organic Anion-Transporting Polypeptide OATP-B. Drug Metabolism and Disposition, 34, 577-582. https://doi.org/10.1124/dmd.105.007872 
Submit or recommend next manuscript to SCIRP and we will provide best service for you:

Accepting pre-submission inquiries through Email, Facebook, LinkedIn, Twitter, etc. A wide selection of journals (inclusive of 9 subjects, more than 200 journals)

Providing 24-hour high-quality service

User-friendly online submission system

Fair and swift peer-review system

Efficient typesetting and proofreading procedure

Display of the result of downloads and visits, as well as the number of cited articles

Maximum dissemination of your research work

Submit your manuscript at: http://papersubmission.scirp.org/

Or contact cm@scirp.org 